Research Article

\title{
Potential Molecular Mechanism of the NPPB Gene in Postischemic Heart Failure with and without T2DM
}

\author{
Yao-Zong Guan, ${ }^{1}$ Rui-Xing Yin $\mathbb{D}^{1},{ }^{1,2,3}$ Guo-Xiong Deng, ${ }^{1}$ Peng-Fei Zheng, ${ }^{1}$ Chun-Xiao Liu, ${ }^{1}$ \\ and Bi-Liu Wei ${ }^{1}$ \\ ${ }^{1}$ Department of Cardiology, Institute of Cardiovascular Diseases, The First Affiliated Hospital, Guangxi Medical University, Nanning, \\ 530021 Guangxi, China \\ ${ }^{2}$ Guangxi Key Laboratory Base of Precision Medicine in Cardio-Cerebrovascular Disease Control and Prevention, Nanning, \\ 530021 Guangxi, China \\ ${ }^{3}$ Guangxi Clinical Research Center for Cardio-Cerebrovascular Diseases, Nanning, 530021 Guangxi, China
}

Correspondence should be addressed to Rui-Xing Yin; yinruixing@163.com

Received 10 May 2020; Revised 24 June 2020; Accepted 11 July 2020; Published 4 August 2020

Academic Editor: Ernesto Greco

Copyright (C) 2020 Yao-Zong Guan et al. This is an open access article distributed under the Creative Commons Attribution License, which permits unrestricted use, distribution, and reproduction in any medium, provided the original work is properly cited.

Background. This study is aimed at investigating natriuretic peptide B (NPPB) coexpression genes and their pathways involved in heart failure (HF) among patients both with and without type 2 diabetes mellitus (T2DM). Methods. The microarray dataset GSE26887, containing 19 postischemic HF patients' peripheral blood samples (7 with T2DM and 12 without T2DM), was examined to detect the genes coexpressed with $N P P B$ using the corr.test function in the R packet. Furthermore, using online analytical tools, we determined the Kyoto Encyclopedia of Genes and Genomes (KEGG) pathway enrichment analysis, Gene Ontology (GO) annotation, and protein-protein interaction (PPI) network of the coexpression genes. The modules and hub genes of the PPI network were then identified using the Cytoscape software. Results. In patients with T2DM, a total of 41 biological processes (BP), 20 cellular components (CC), 13 molecular functions (MF), and 41 pathways were identified. Furthermore, a total of $61 \mathrm{BPs}, 16 \mathrm{CCs}, 13 \mathrm{MFs}$, and 22 pathways in patients without T2DM were identified. In both groups of patients, 17 BPs, 10 CCs, 6 MFs, and 13 pathways were enriched. We also identified 173 intersectional coexpression genes (63 positively, 106 negatively, and 4 differently coexpressed in patients with and without T2DM, respectively) in both types of patients, which were enriched in 16 BPs, 8 CCs, 3 MFs, and 8 KEGG pathways. Moreover, the PPI network (containing 237 edges and 170 nodes) with the top module significantly enriched in 4 BPs (tricarboxylic acid metabolic process, citrate metabolic process, tricarboxylic acid cycle, and aerobic respiration) and 3 pathways (citrate cycle, malaria parasite metabolic pathway, and AGE-RAGE signaling pathway in diabetic complications) was constructed. DECR1, BGN, TIMP1, VCAN, and CTCF are the top hub genes. Conclusions. Our findings may elucidate the functions and roles of the NPPB gene in patients with postischemic HF and facilitate HF management.

\section{Introduction}

Heart failure (HF) is a challenge for numerous cardiovascular specialists, as it affects both the health and quality of life of a tremendous number of patients. It is estimated that 26 million people worldwide suffer from HF, according to data from a prior survey [1]. Moreover, annual costs to treat and manage HF ranges from International Dollars (Int\$) $2,496.00$ to Int $\$ 84,434.00$ per patient [2]. However, it is estimated that the in-hospital mortality ranges from $4 \%$ to $30 \%$ and that the all-cause 1-year mortality rates among patients with acute HF and patients with chronic HF were 23.6\% and $6.4 \%$, respectively [3]. The etiology of heart failure involves coronary artery disease, rheumatic heart disease, cardiomyopathies, hyperthyroidism, and so on. Among these diseases, ischemic heart failure is common, especially when caused by ST-segment elevation myocardial infarction [4]. Although the numbers of chest-pain centers and cardiac care units (CCUs) have been increasing and thus more patients have received timely and effective interventions, 


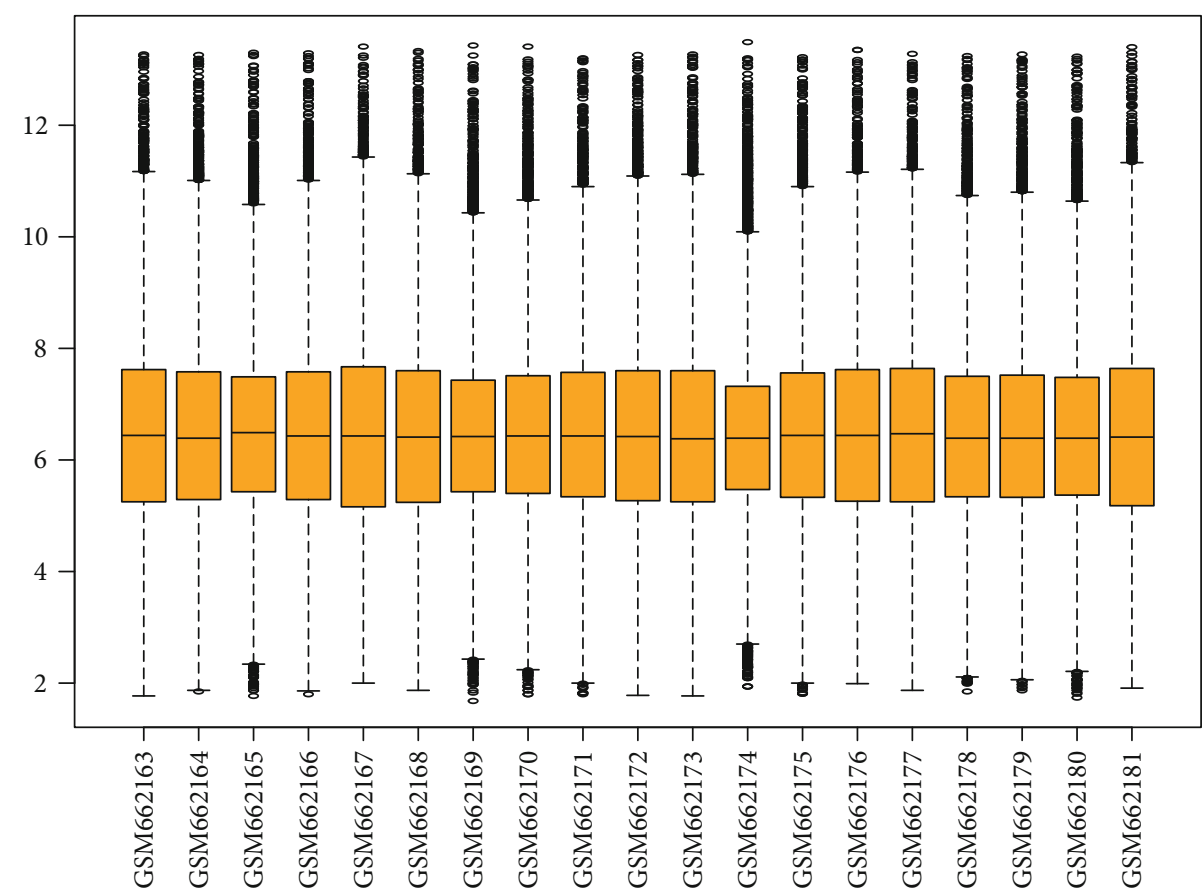

Figure 1: Normalization of gene expression. The orange box represents the expression of genes, and the black line in the box represents the median. The $x$-axis represents the sample name, and the $y$-axis represents the expression level.

postischemic HF remains a challenge that cannot be neglected any further.

Type 2 diabetes mellitus (T2DM), which is an endocrine disease that mainly leads to vascular and nerve damage, is regarded as an equal-risk syndrome of coronary heart disease and accompanies patients for the rest of their lives [5]. It is reported that T2DM not only promotes the development of HF but also increases the risk of cardiovascular disease (CVD) 2- to 4 -fold [6, 7]. It is estimated that more than 400 million persons are affected by T2DM worldwide, costing $\$ 1.3$ trillion annually [8]. In addition, the coexistence of $\mathrm{HF}$ and $\mathrm{T} 2 \mathrm{DM}$ is common, and in populations ranging from 33 years to 84 years, the prevalence of $\mathrm{HF}$ in people with T2DM was $12 \%$ [9]. Moreover, T2DM and HF mutually promote the development of each other, and it is more complicated to treat HF patients with T2DM [10].

Currently, the diagnosis of HF is mainly based on clinical manifestations. Fortunately, serum BNP (encoded by the $N P P B$ gene) and NT-pro-BNP levels have greatly contributed to the proper diagnosis of HF [11]. BNP is mainly secreted by atrial myocytes and thus reflects the heart load. BNP can represent powerful biological effects, such as natriuresis, vasodilation, myocardial apoptosis inhibition, and modulation of immune and inflammatory responses of cardiac injury [12-14]. Some prior studies suggest that BNP can be used as a biomarker for prognosis in patients with HF, and it also participates in both occurrence and development of T2DM and ischemic cardiomyopathy $[15,16]$. What's more, in diabetic patients, BNP can be used for screening the absence of left ventricular dysfunction [17]. Therefore, BNP is associated with HF as well as T2DM. However, results from early researches show us that serum BNP levels are higher in HF patients with diabetes than in HF patients without diabetes, while some others report the opposite result $[18,19]$. It is not yet clear whether there are some shared and specific mechanisms of the $N P P B$ gene in HF patients with and without T2DM.

In recent years, microarray sequencing technology has rapidly developed and has significantly assisted basic and clinical medicine. The Gene Expression Omnibus (GEO) database is a huge repository that stores a series of highthroughput microarray and next-generation sequence functional genomic datasets and is free for global researchers to use for mining purposes [20]. In this study, we aimed to further understand the function of the NPPB gene in HF patients and better inform HF management by detecting the $N P P B$ coexpression genes and pathways enriched in patients with postischemic HF either with or without T2DM.

\section{Methods}

2.1. Affymetrix Microarray Data. The microarray dataset GSE26887 was retrieved from the GEO database. This dataset contained 24 samples from 19 patients with postischemic heart failure (7 with T2DM and 12 without T2DM) and 5 from control nonfailing hearts [21]. We recruited patients with postischemic heart failure either with T2DM (DHF group, $n=7$ ) or without T2DM (nDHF group, $n=12$ ) for analysis. The extracted data were normalized before further analysis in order to ensure the comparability of samples by the limma package that is available in the $\mathrm{R}$ platform [22] (Figure 1).

2.2. Identification of $N P P B$ Coexpression Genes. A screening of coexpression genes for $N P P B$ from the samples was performed by the corr.test function in $\mathrm{R}$ (version 3.6.1). 
Screening criteria were as follows: $P<0.05$ and $\mid$ Pearson correlation coefficient $\mid \geq 0.4$. The online analytical tool Draw Venn Diagram (http://bioinformatics.psb.ugent.be/ webtools/Venn/) was then used to determine the intersectional coexpression genes of both groups.

2.3. GO and KEGG Pathway Enrichment Analyses. The online database DAVID (version 6.8) [23] was used for GO and KEGG enrichment analyses [24, 25]. A $P$ value of $<0.05$ was set as significant. The ggplot2 package was used for the visualization of the results in $\mathrm{R}$ (version 3.6.1).

2.4. Integration of the PPI Network. The STRING (version 10.5) database was used for evaluating the interactions among the coexpression genes, and a combined interaction score of $>0.4$ was set as significant [26]. In addition, the top 10 hub genes were identified using Cytoscape plugin cytoHubba (version 0.1) with the degree ratio ranking method. Furthermore, the MCODE and ClueGO apps in Cytoscape were used to identify the modules, namely the GO annotation and KEGG pathway enrichment analyses, respectively, of the PPI network [27].

\section{Results}

3.1. Identification of NPPB Coexpression Genes. A total of 577 negatively coexpressed genes and 457 positively coexpressed genes in the DHF group were identified, along with 666 negatively coexpressed genes and 422 positively coexpressed genes in the nDHF group. Figure 2 portrays 106 negatively and 63 positively coexpressed genes in both patient types, whereby 173 intersectional coexpression genes were screened out. Interestingly, of these intersectional coexpression genes, we found 3 genes (CENPBD1P1, KHDRBS3, and PHOX2B) that were positively coexpressed with $N P P B$ in patients with T2DM, but negatively coexpressed in patients without T2DM, and 1 gene (NQO1) that was negatively coexpressed with $N P P B$ in patients with T2DM, but positively coexpressed in patients without T2DM.

3.2. Functional $G O$ and KEGG Pathway Enrichment Analyses. GO analyses revealed $41 \mathrm{BPs}, 20 \mathrm{CCs}$, and 13 MFs in the DHF group, and $61 \mathrm{BPs}, 16 \mathrm{CCs}$, and $13 \mathrm{MFs}$ in the nDHF group (details in Tables S1 and S2). Due to the excessive number of enrichment analyses, the top seven BPs, CCs, and MFs were selected for visualization with $P<$ 0.05 (Figures 3(a) and 3(b)). Furthermore, there were 10 BPs (fatty acid beta-oxidation, oxidation-reduction process, metabolic process, mitochondrial respiratory chain complex I assembly, glyoxylate metabolic process, ubiquinone biosynthetic process, positive regulation of cell growth, tricarboxylic acid cycle, cell adhesion, and aerobic respiration), $8 \mathrm{CCs}$ (mitochondrial inner membrane, extracellular space, mitochondrion, extracellular matrix, myelin sheath, extracellular exosome, $\mathrm{Z}$ disc, and mitochondrial matrix), and $3 \mathrm{MFs}$ (growth factor activity, protein binding, and electron carrier activity) enriched in both patient groups. There were 41 identified pathways in patients with T2DM (Figure 4(a)) and 22 in patients without T2DM (Figure 4(b)) (details in Tables S1 and S2). Moreover, common pathways are shown in Table 1.

The analyses further identified $16 \mathrm{BPs}, 8 \mathrm{CCs}$, and $3 \mathrm{MFs}$ that were enriched by intersectional coexpression genes in both patient groups (Figure 3(c)), and these genes mainly clustered in the following 8 pathways: the citrate cycle (TCA cycle), carbon metabolism, biosynthesis of antibiotics, malaria, glyoxylate metabolism, dicarboxylate metabolism, cardiac muscle contraction, and African trypanosomiasis (Figure 4(c)) (details in Table S3).

3.3. PPI Network Construction and Hub Gene Identification. As Figure 5 shows, the interactions among intersectional coexpression genes were displayed by a PPI network with 273 edges and 170 nodes. This finding was saved in TSV format and then imported into Cytoscape for visualization. With a cutoff criterion of a degree that is $>5$ and a $\mathrm{K}$ - core $>5$, only one module with 4 BPs (tricarboxylic acid metabolic process, citrate metabolic process, tricarboxylic acid cycle, and aerobic respiration) and 3 pathways (citrate cycle, malaria parasite metabolic pathway, and AGE-RAGE signaling pathway in diabetic complications) significantly enriched was identified. With the degree ratio ranking method, the top 10 hub genes of this PPI network were also identified (CS, DECR1, ACO2, BGN, TIMP1, CTGF, VCAN, SERPINE1, $S D H C$, and CCL2). With the same cutoff criterion, a PPI network that consists of 953 nodes and 4,946 edges of $N P P B$ coexpression genes in the DHF group, and a PPI network of 1,009 nodes and 4,245 edges in the nDHF group were also constructed. The top 10 hub genes of the former were $C Y C S$, FN1, CS, DECR1, ACO2, ATP5A1, NDUFAB1, EGF, ATP5H, and $A T P 5 C 1$, while the top 10 hub genes of the latter were $C S$, DECR1, BGN, TIMP1, ACO2, CTGF, VCAN, SERPINE1, CCL2, and SDHC (Figures 6(a) and 6(b), respectively). The visualization of these two PPI networks and their modules were concluded in Supplementary Materials (Figures S1-S4).

3.4. Verification of Hub Genes. Another dataset, GSE5406, containing 210 left ventricular myocardium samples (86 with idiopathic dilated cardiomyopathy, 108 with ischemic cardiomyopathy, and 18 unused donor hearts) was downloaded from the GEO database to verify the hub genes. We selected the heart failure with advanced ischemic cardiomyopathy samples $(n=108)$ for NPPB coexpression gene analysis with the same method as described in Methods. The correlation values of the NPPB coexpression gene in the GSE5406 dataset and in the GSE26887 dataset are shown in Table 2. Except for CCL2, other hub genes are coexpressed to $N P P B$ with $P<0.05$ and $\mid$ Pearson correlation coefficient $\mid>0.2$. Both the positive coexpressed relationship and negative coexpressed relationship correspond.

\section{Discussion}

Although living and medical standards have undergone remarkable progress, heart failure remains a worldwide challenge, which costs countries a tremendous amount of money and affects the quality of life of patients at different degrees. Ischemic cardiomyopathy is one of the most common causes 


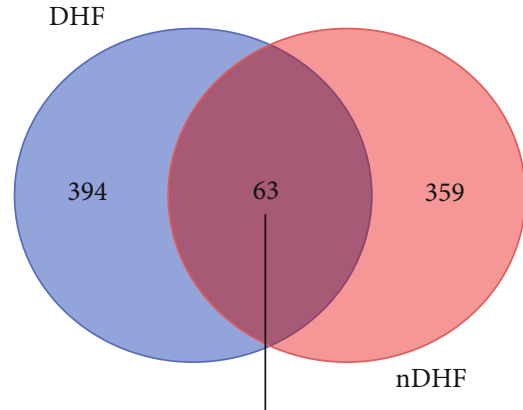

TAGLN/RN7SK/CCNA1/TGFB2/KCNT2/TMED3/TNC/ MFAP2/ZPR1/CLIC1/SERPINE2/PDLIM7/PXDC1/ID4/ SCG2/ITGBL1/PODN/F2RL1/JUNB/CCL2/SLC39A13/ GLIS3/RGS4/AEBP1/THBS2/PRSS23/PRAF2/PAPPA/ FAM104A/HTRA1/SEC61B/RUNX1/SCRG1/PTGFR/ CYSTM1/NPPA/SERPINE1/COX6A1/C11 orf24/IER3/ BMP8A/EGR2/CCN1/ETV5/VCAM1/CCN2/SNCAIP/ AUP1/FSTL3/CCN3/MGP/CPT1C/PLPPR4/PCOLCE/ ARPC3/SOCS3/TIMP1/BGN/MIR21/FAM120AOS/ PRDM7/VCAN/IFT20

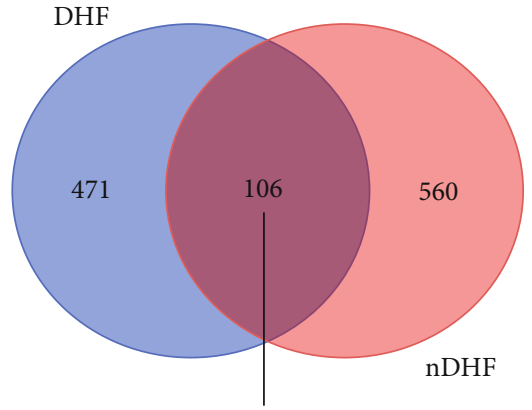

DECR1/PCMTD2/CLGN/PDE1C/LDHD/DSP/ECH1/CS/ ANXA3/ACAT1/TNNT2/COQ9/RBFOX1/TMEM143/ TIMM22/CKMT2/STRIP2/AQP4/COX10/MTTP/ACO2/ ACOT2/NDUFS2/SLC35F1/TLL2/FHL2/DPYSL4/CD38/ PCYOX1/EGFLAM/FGF12/FAM78A/PTER/ATP5F1A/ SLC48A1/PDE3A/KY/PFKM/DCUN1D2/PRXL2A/CA8/ PPP1R3A/LRRC39/ALDH5A1/DMAC2/MCCC2/RYR2/ ASB15/CCDC68/PTDSS1/PPP1R12B/MAOB/PLCB4/ CD99L2/ST13P4/MRPL46/KCNAB2/UPB1/EPM2A/ LPCAT4/PKD1L1/ANKRD2/COQ8A/TMEM109/AGL/ ANP32C/SLC2A4/NIPAL3/KLHL31/CACNB2/HOOK1/ PAIP2B/CMBL/SDHC/DDX1/ALAS1/SMCO1/PYGM/ FAM219B/SLC5A1/MRPS25/FAM107A/EIF4H/NFS1/ CCDC85A/NDUFA9/PEX19/SPHKAP/DDO/AIG1/SIAE/ GPRC5B/CEACAM1/AQP7/TMEM185A/BCO2/RNLS/ NXPE3/PDHB/TADA2B/NCOA4/GALNT16/SELENBP1/ AMER1/TRIM68/SLC41A1

(b) Negatively co-expression genes

(a) Positively co-expression genes

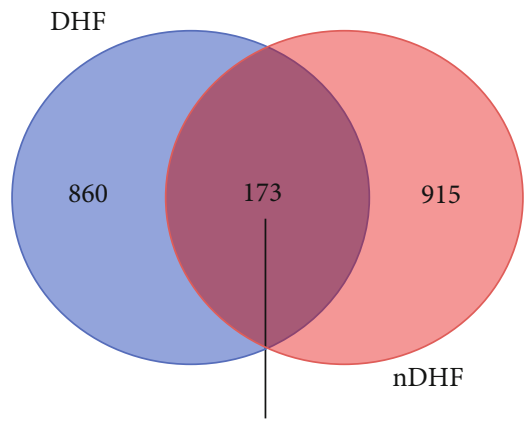

\begin{tabular}{|l|}
\hline CCNA1/DECR1/TGFB2/PCMTD2/CLGN/PDE1C/ECH1/DSP/ \\
PCOLCE/LDHD/BCO2/PDLIM7/ANXA3/ACAT1/CD38/PODN/ \\
F2RL1/COQ9/RBFOX1/TMEM143/CS/TIMM22/RGS4/PPBP/ \\
STRIP2/PRAF2/AQP4/COX10/PAPPA/ARPC3/RUNX1/SCRG1/ \\
MTTP/PTGFR/ACO2/PHOX2B/CYSTM1/NPPA/ACOT2/RCN3/ \\
NDUFS2/CCN2/IER3/SLC35F1/TLL2/BMP8A/EGR2/FHL2/ \\
SNCAIP/DPYSL4/TNNT2/PCYOX1/EGFLAM/FGF12/FAM78A/ \\
PTER/KY/ATP5F1A/SLC48A1/PDE3A/MGP/PFKM/TMED3/ \\
CENPBD1P1/DCUN1D2/SOCS3/TIMP1/PRXL2A/BGN/RNLS/ \\
FAM120AOS/MIR21/ALAS1/PPP1R3A/CLIC1/CCL2/TRIM68/ \\
FAM104A/LRRC39/ALDH5A1/DMAC2/MCCC2/ANP32C/ \\
ASB15/CCDC68/PTDSS1/COX6A1/RN7SK/TAGLN/KCNT2/ \\
PPP1R12B/MAOB/SERPINE2/PXDC1/PLCB4/SCG2/TNC/ \\
CD99L2/ITGBL1/ST13P4/NQO1/MRPL46/KCNAB2/AMER1/ \\
UPB1/JUNB/AGL/SLC39A13/GLIS3/KHDRBS3/LPCAT4/ID4 \\
PKD1L1/AEBP1/ANKRD2/COQ8A/THBS2/TMEM109/AQP7/ \\
PRSS23/EPM2A/RYR2/MFAP2/SLC2A4/NIPAL3/HTRA1/ \\
KLHL31/CACNB2/NFS1/PAIP2B/CMBL/SDHC/DDX1/CA8/ \\
SERPINE1/SMCO1/VCAN/C11orf24/VCAM1/SELENBP1/ \\
CCN1/ETV5/FAM219B/AUP1/FSTL3/HOOK1/MRPS25/DDO/ \\
SLC5A1/EIF4H/FAM107A/CCDC85A/NDUFA9/TMEM185A/ \\
SPHKAP/CCN3/AIG1/GPRC5B/CPT1C/CEACAM1/GALNT16/ \\
PLPPR4/CKMT2/PYGM/IFT20/NXPE3/SEC61B/PEX19/SIAE/ \\
PDHB/TADA2B/NCOA4/PRDM7/SLC41A1
\end{tabular}

(c) Co-expression genes

FIGURE 2: Venn diagram of coexpression genes. (a) Venn diagram of genes positively coexpressed with NPPB. (b) Venn diagram of genes negatively coexpressed with $N P P B$. (c) Venn diagram of $N P P B$ coexpression genes. DHF: postischemic patients with T2DM. nDHF: postischemic patients without T2DM. 


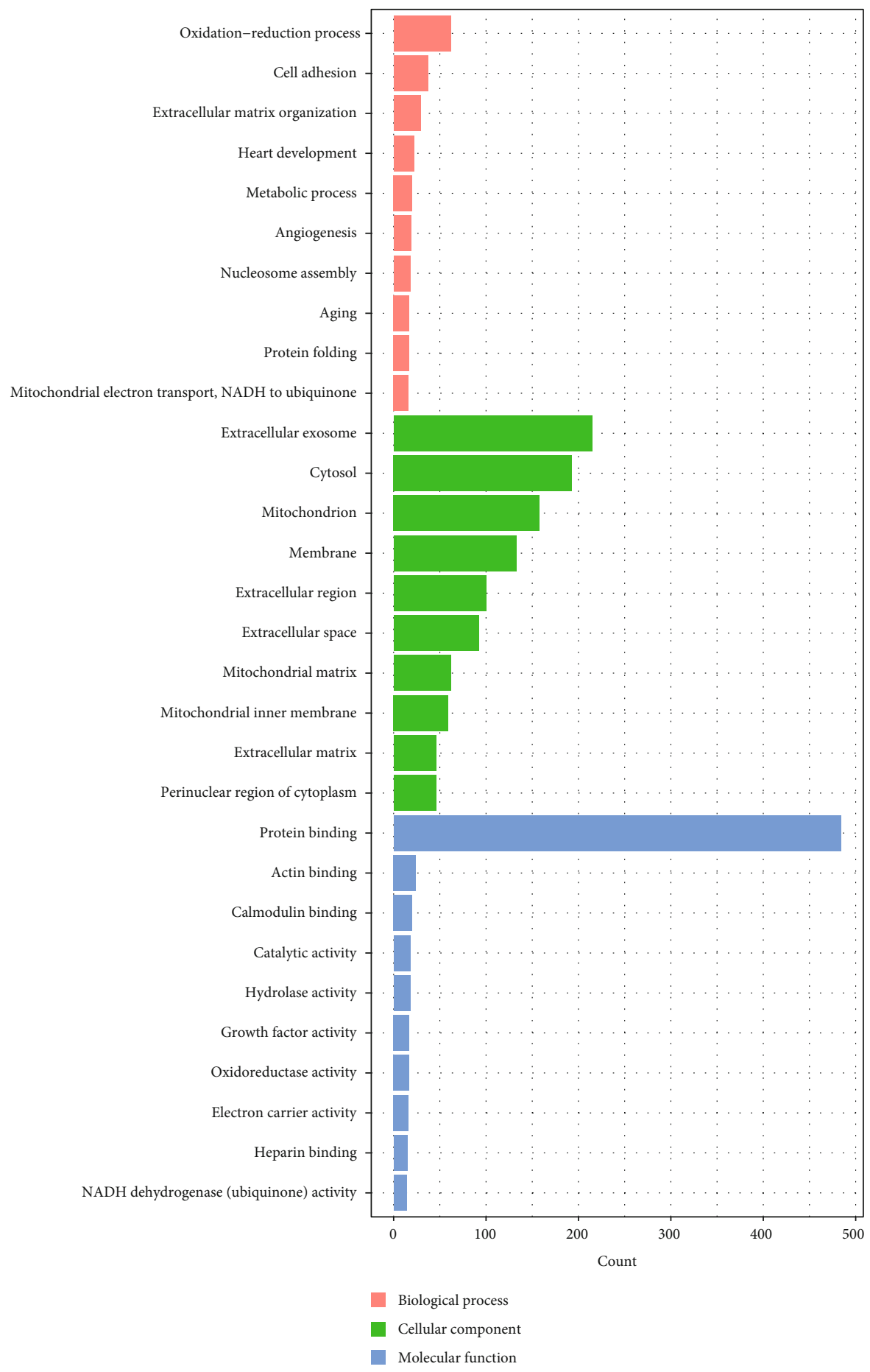

(a)

Figure 3: Continued. 


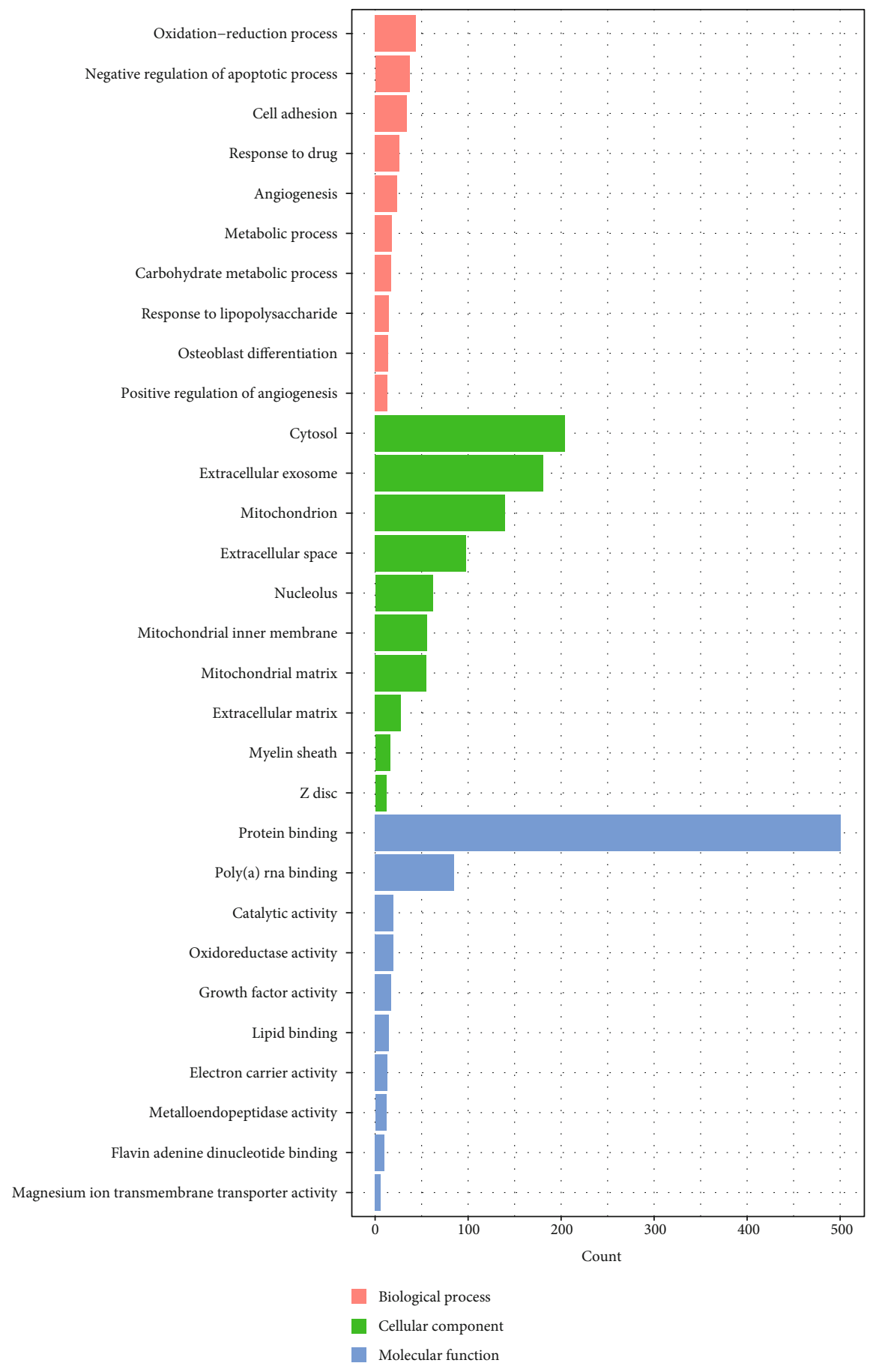

(b)

Figure 3: Continued. 


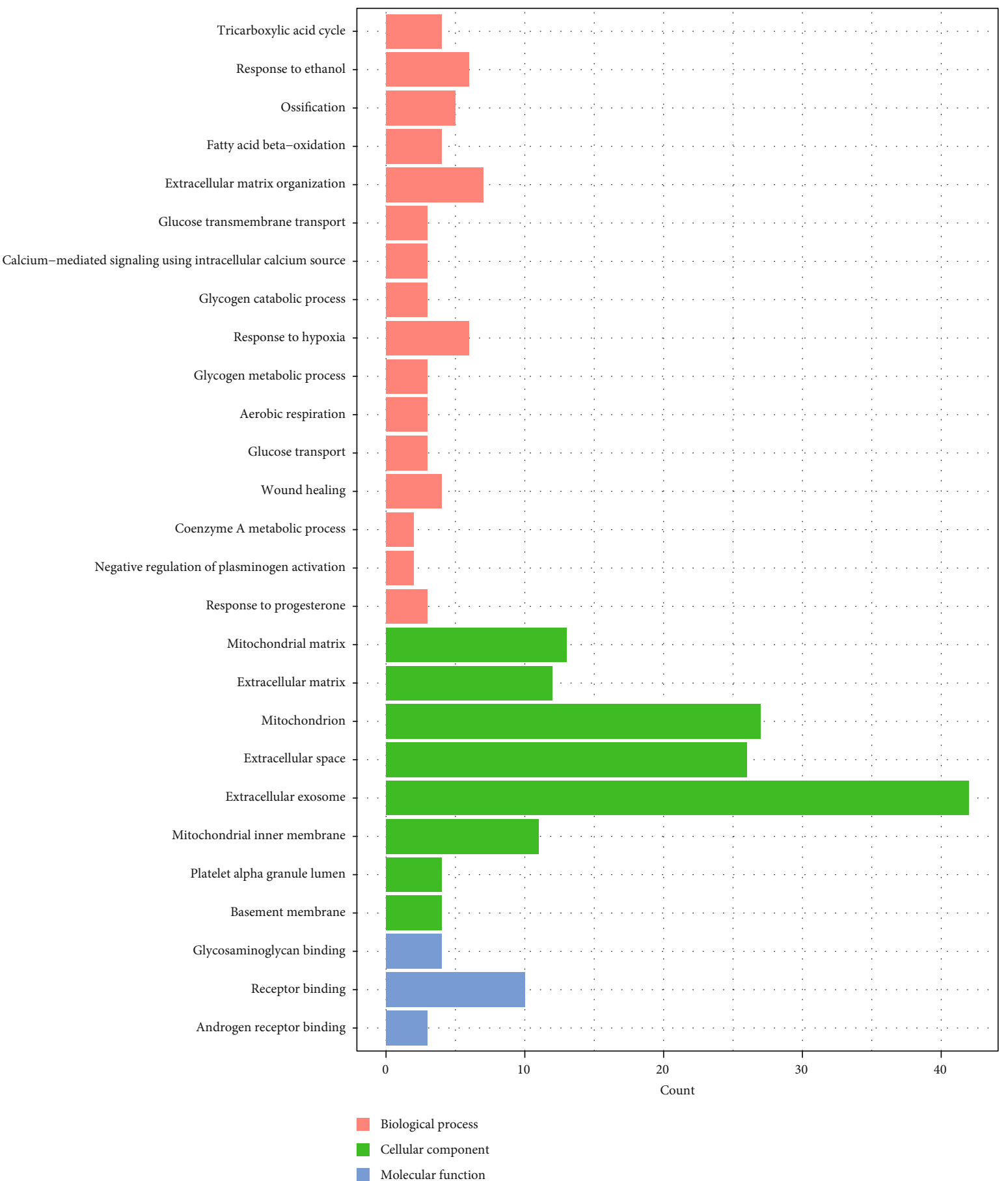

(c)

FIGURE 3: GO annotation of NPPB coexpression genes in postischemic heart failure patients: (a) in postischemic patients with T2DM and (b) in postischemic patients without T2DM. (c) Based on the intersectional coexpression genes of the two types of patients.

of heart failure; moreover, a portion of these patients also suffer from other diseases, such as type 2 diabetes mellitus, which complicates the treatment interventions for heart failure. Angiotensin-converting enzyme inhibitors, betablockers, diuretics, positive inotropic drugs, and cardiac resynchronization therapy (CRT) have been widely used in postischemic heart failure therapy, but quite a few patients inevitably go into end-stage heart failure for a variety of rea- sons [8]. Thus, they experience repeated hospitalizations, a severe decline in quality of life, complications in other organs, and even death. Serum BNP, encoded by NPPB, is secreted primarily by atria muscle cells, and BNP level increases when the heart is overloaded. It has been applied in clinics as a diagnostic and prognostic biomarker of HF for a long time, which is a great achievement [28]. Besides, $\mathrm{BNP}$ is also reportedly associated with the development of 


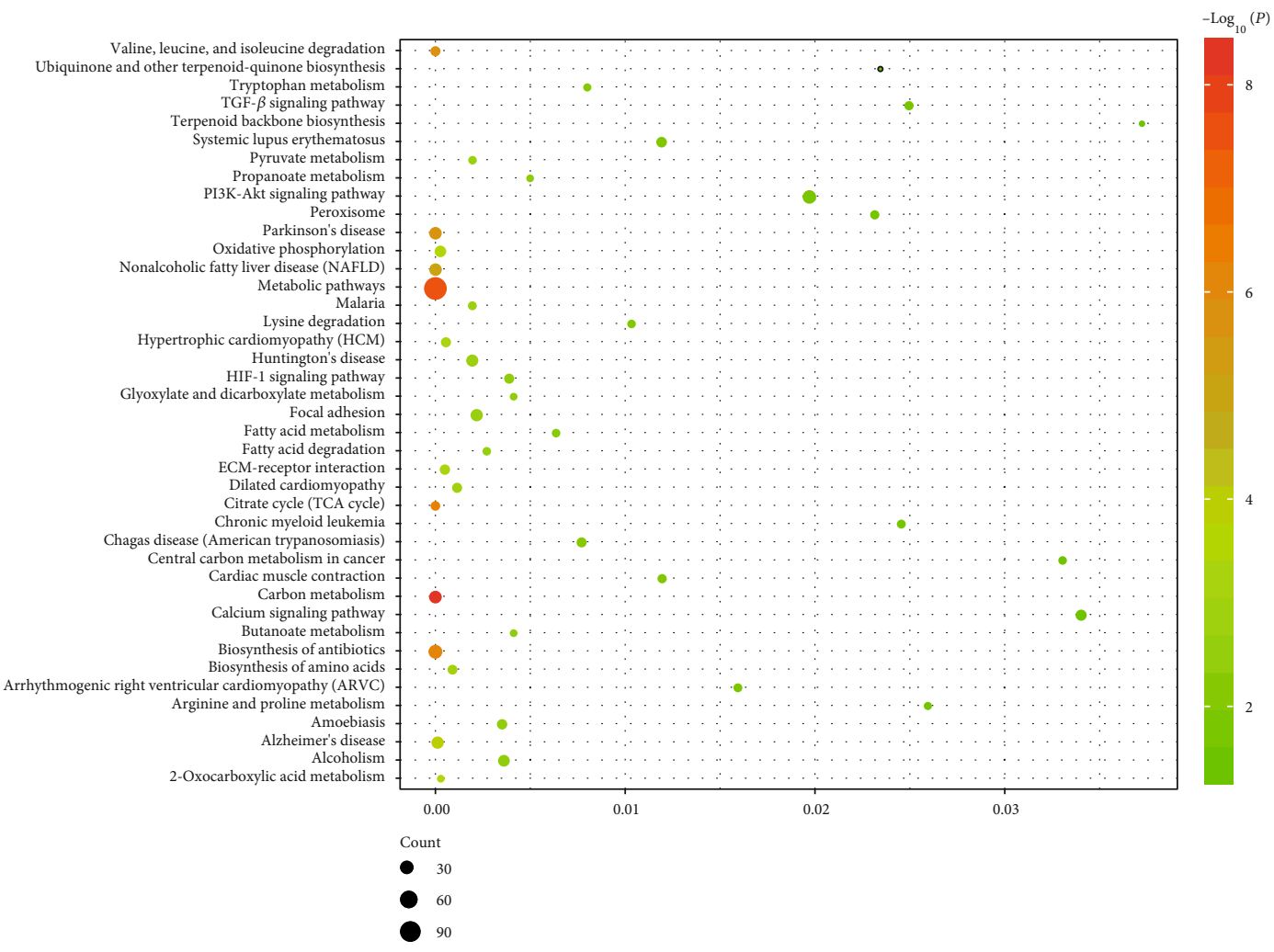

(a)

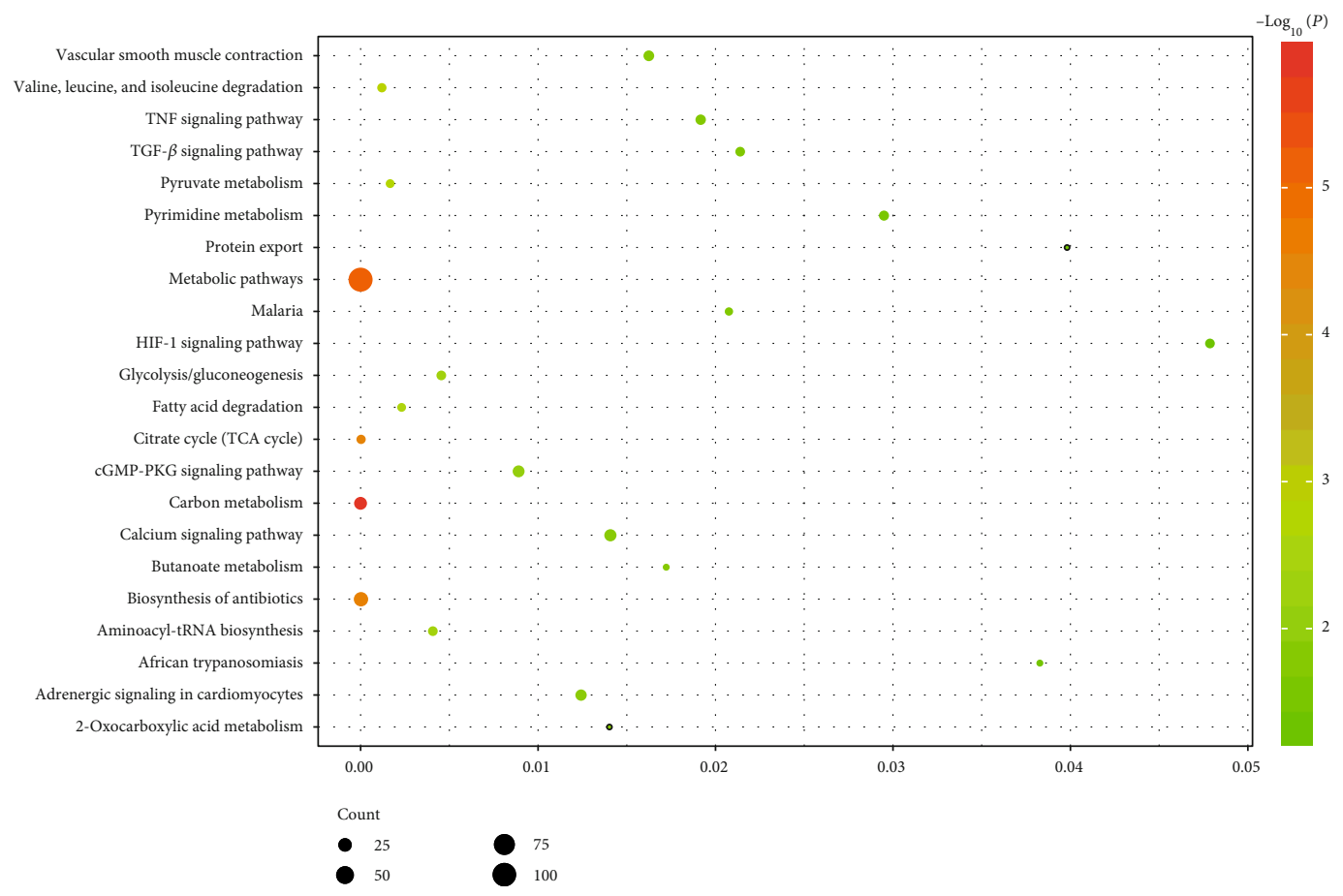

(b)

Figure 4: Continued. 


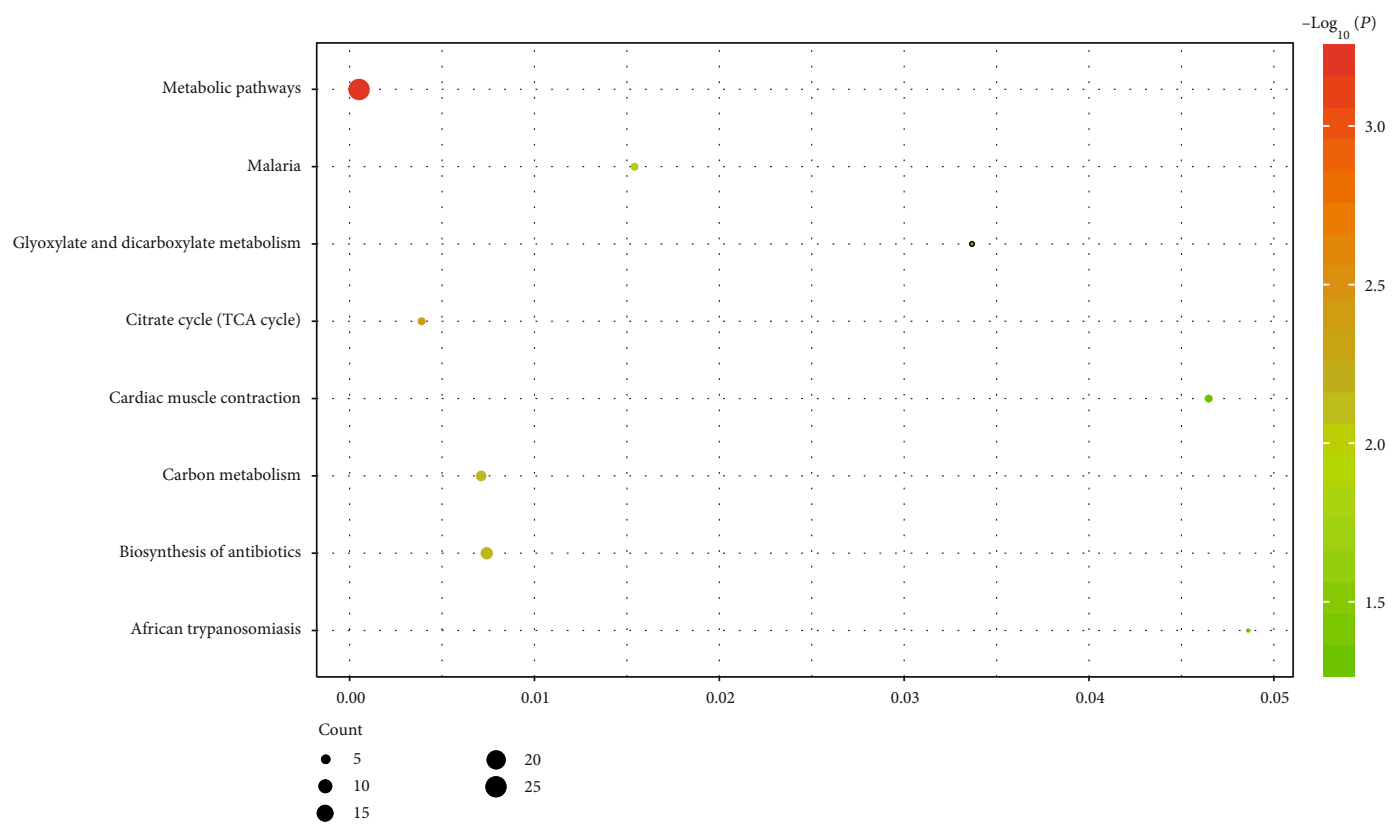

(c)

FIgURE 4: KEGG pathways of NPPB coexpression genes in postischemic heart failure patients: (a) in postischemic patients with T2DM and (b) in postischemic patients without T2DM. (c) Based on the intersectional coexpression genes of the two types of patients.

T2DM, and in turn, diabetes affected its expression in patients with HF. Some early researches reveal that the serum BNP level in HF patients without diabetes is higher than that in HF patients with diabetes, while other researches report the opposite result. Up to now, the mechanism is still completely clear. In this study, $N P P B$ coexpression genes and their GO and KEGG pathways were identified in postischemic HF with T2DM and without T2DM, respectively, in order to further understand the potential mechanism of $N P P B$ in postischemic HF patients with and without T2DM.

Heart failure is the result of the contradiction between the supply and demand of oxygen, blood, and energy, and the tricarboxylic acid cycle (TCA cycle) and mitochondrial respiratory transport chain are important links in glycolysis. As screened by the Venn diagram, a total of 63 positively coexpressed genes were identified. Carnitine palmitoyl transferase 1 (CPT1) encodes an important enzyme in the body, involved in fatty acid metabolism. As a subtype of CPT1, CPT1C can promote cell survival under metabolic stress conditions [29]. Furthermore, HtrA serine peptidase 1 (HTRA1) encodes a protein that is suggested to be a cell growth regulator, and its loss impairs smooth muscle cell maturation [30]. In a previous research, hypermethylation of the SOCS3 gene could be an underlying mechanism of intimal hyperplasia and restenosis. SOCS3 can also regulate cavin-1 function by enhancing its stability and consequently maintaining expression levels of caveolin-1 and cell surface caveolae. Moreover, proteins encoded by cavin- 1 are also believed to modify lipid metabolism and insulin-regulated gene expression [31, 32]. In terms of vascular function, CCN1 not only functions as an inhibitory regulator of SMC muscle contractility through inhibiting actomyosin interactions but also regulates TNF- $\alpha$ induced vascular endothelial cell apoptosis [33]. The
PDLIM7 gene product is involved in actin filamentassociated complex assembly, which is essential for the transmission of ret/ptc2 mitogenic signaling. In addition, its expression is positively correlated to typical smooth muscle cell markers in atherosclerosis plaques, and PDLIM7 silencing in vitro led to downregulation of smooth muscle cell (SMC) markers, disruption of actin cytoskeleton, decreased cell spreading, and increased proliferation [34]. The data from Thomsen et al. suggested that in patients with ischemic heart disease, increased plasma MGP levels are indicative of a progressing calcification process [35]. Moreover, proteaseactivated receptor $2(P A R 2)$ in microvascular endothelial cells is indispensable for vascular stability, and its deficiency attenuates atherosclerosis [36, 37]. The abovementioned genes mainly play a role in energy supply and metabolism, cell proliferation and apoptosis, and vessel function and development, and they have been reportedly associated with blood and oxygen supply and cardiac remodeling in patients with HF.

On the other hand, a Venn diagram allowed identifying 106 genes negatively coexpressed with $N P P B$. Coq $8 p$ and human COQ8A are related to CoQ biosynthesis, and acute inhibition of Coq8p is sufficient to cause CoQ deficiency and respiratory dysfunction [38]. NDUFS2 and NDUFA9 encode compound I subunits in the mitochondrial membrane respiratory chain, while SDHC encodes compound II subunits. Also, DECR1 encodes an enzyme, referred to as $\mathrm{NADPH}$, which provides $\mathrm{H}^{+}$ions for $\mathrm{NAD}^{+}$and then converts to $\mathrm{NADH}$ to participate in the respiratory chain. In addition to the respiratory chain, the TCA cycle also features several genes that are mainly active in its processes [39]. $P D H B$ encodes a pyruvate dehydrogenase compound, which catalyzes the conversion of pyruvate into acetyl-CoA and 


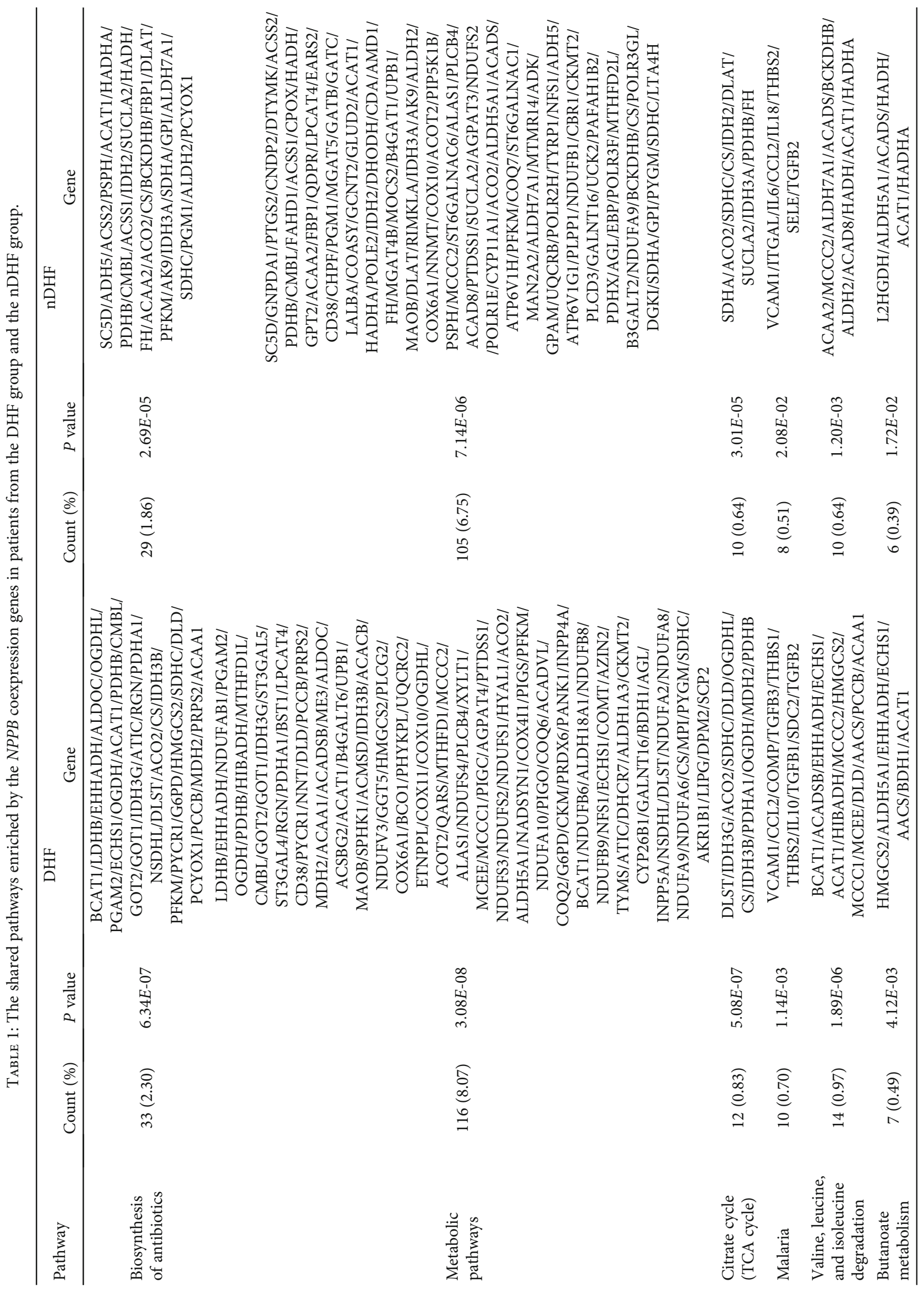




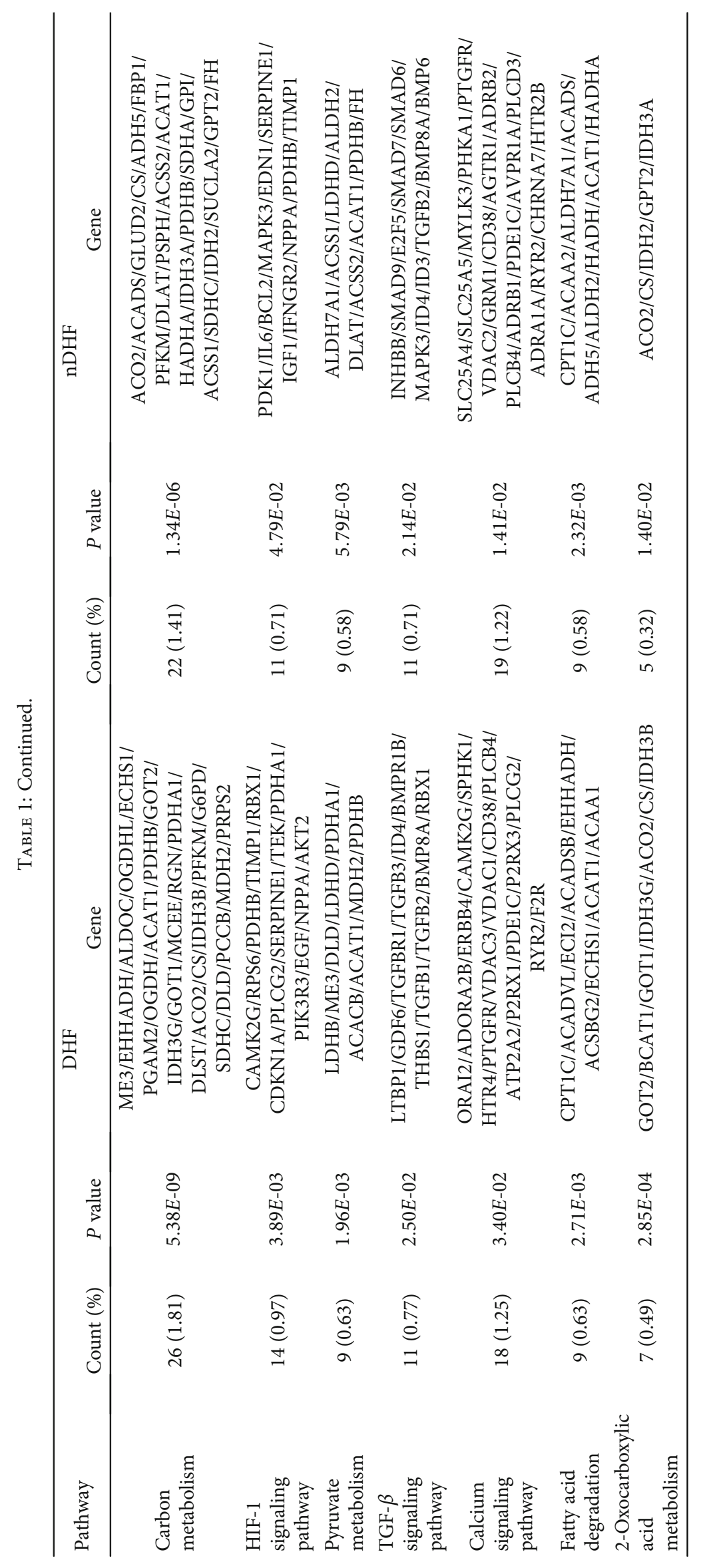




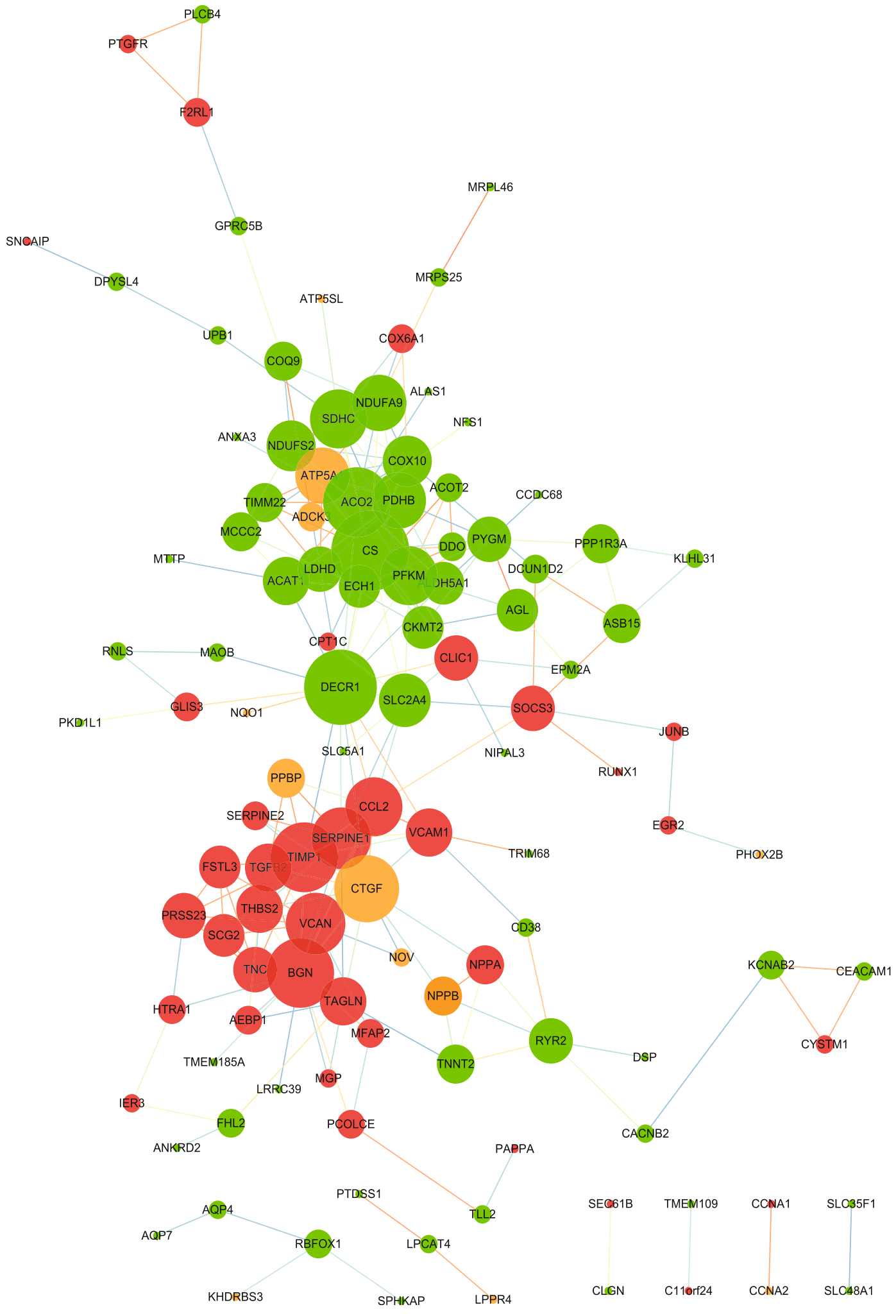

(a)

Figure 5: Continued. 


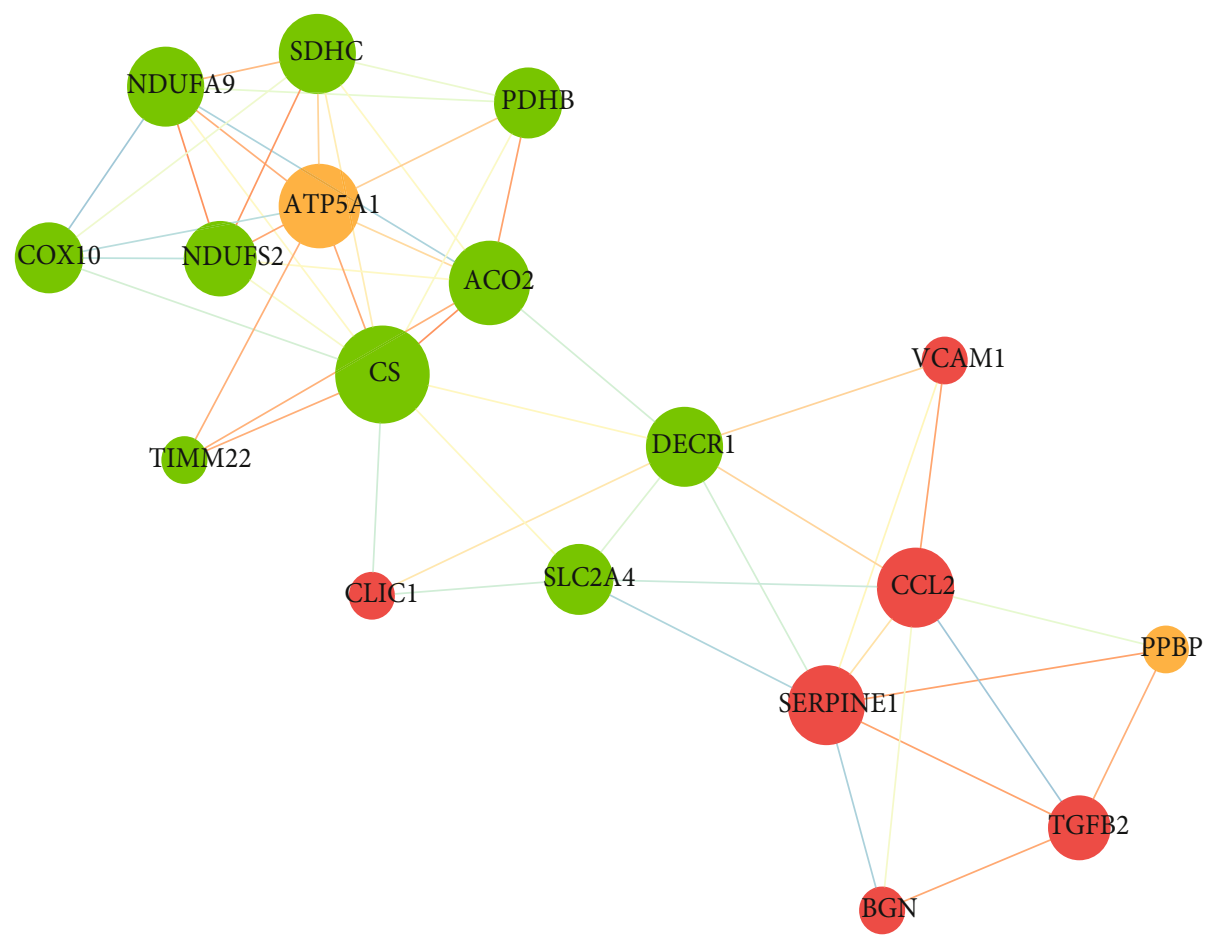

(b)

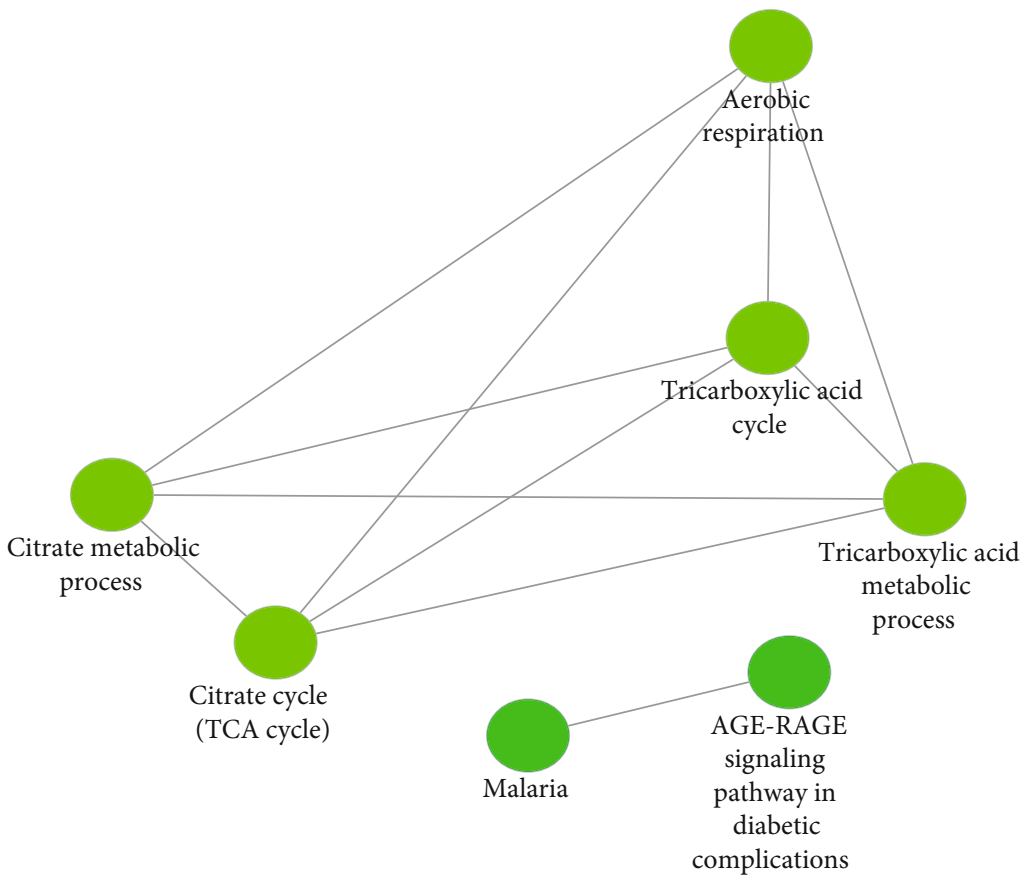

(c)

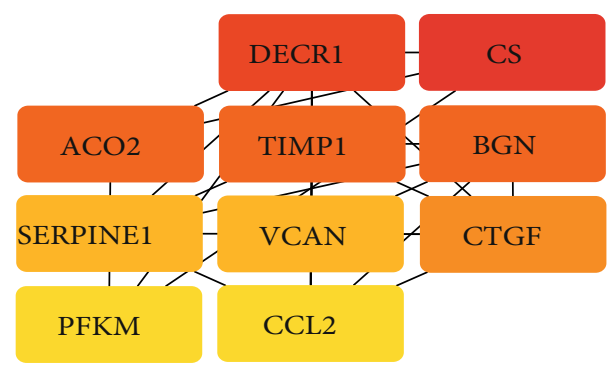

FIGURE 5: Protein-protein interaction (PPI) network of intersectional NPPB coexpression genes. (a) PPI network based on the intersectional $N P P B$ coexpression genes of two types of patients. The red ball represents positive coexpression, while the green ball represents negative coexpression. The thickness of the line represents the strength of the correlation. (b) Module identified with a cutoff criterion of MCODE score $>5$. (c) Biological process and KEGG pathways enriched in the module. (d) Top 10 hub genes. The color depth represents the ranking of hub genes. The sequence of colors is red-orange-yellow from high ranking to low ranking.

carbon dioxide for the TCA cycle. Citrate synthase, which is encoded by CS, catalyzes citric acid synthesis from oxaloacetic acid and acetyl-CoA; furthermore, citric acid synthesis by oxaloacetic acid and acetyl-CoA is catalyzed by cisaconitum, which is encoded by ACO2. ALAS1 encodes mitochondrial enzymes that catalyze rate-limiting steps in the heme (iron protoporphyrin) biosynthesis pathway. In the context of cell proliferation and vascular function, Yan reported that in 


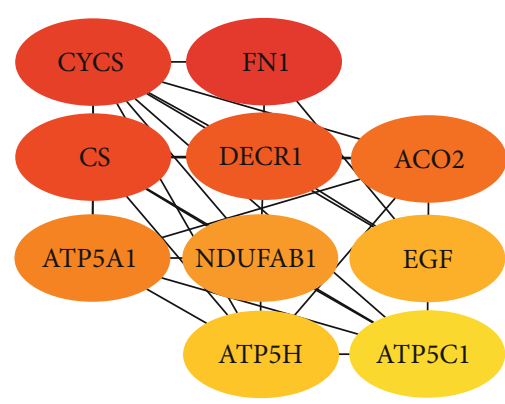

(a)

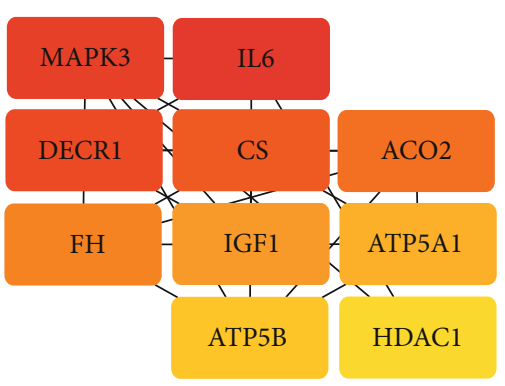

(b)

Figure 6: Top 10 hub genes of the PPI network. (a) Top 10 hub genes of the PPI network based on the NPPB coexpression genes in the DHF group. (b) Top 10 hub genes of the PPI network based on the NPPB coexpression genes in the nDHF group. The sequence of colors is redorange-yellow from high ranking degree to low ranking degree.

TABle 2: Verification of the hub genes.

\begin{tabular}{lcccccc}
\hline Hub gene & $\begin{array}{c}\text { Cor } \\
\text { GSE5406 }\end{array}$ & $\begin{array}{c}\text { Cor } \\
\text { GSE26887 }\end{array}$ & $\begin{array}{c}P \\
\text { DHF }\end{array}$ & $\begin{array}{c}\text { Cor } \\
\text { GSE26887 }\end{array}$ & $\begin{array}{c}P \\
\text { nDHF }\end{array}$ \\
\hline DECR1 & -0.48 & $<0.01$ & -0.83 & 0.02 & -0.74 & $<0.01$ \\
BGN & 0.51 & $<0.01$ & 0.87 & 0.01 & 0.59 & 0.04 \\
TIMP1 & 0.44 & $<0.01$ & 0.89 & $<0.01$ & 0.76 & $<0.01$ \\
VCAN & 0.50 & $<0.01$ & 0.81 & 0.03 & 0.61 & 0.04 \\
CTCF & 0.50 & $<0.01$ & 0.95 & $<0.01$ & 0.79 & $<0.01$ \\
CS & -0.35 & $<0.01$ & -0.84 & 0.02 & -0.60 & 0.04 \\
ACO2 & -0.34 & $<0.01$ & -0.86 & 0.01 & -0.62 & 0.03 \\
SERPINE1 & 0.38 & $<0.01$ & 0.89 & $<0.01$ & 0.68 & 0.01 \\
SDHC & -0.21 & 0.03 & -0.92 & $<0.01$ & -0.64 & 0.03 \\
CCL2 & 0.08 & 0.39 & 0.85 & 0.02 & 0.69 & 0.01 \\
\hline
\end{tabular}

Cor: Pearson correlation coefficient.

senescent vascular SMCs, PDE1A and PDE1C mRNA levels are significantly upregulated, and cellular senescent makers were reduced when PDE1 was inhibited [40]. Data from Begum et al. suggest that therapies specifically aimed at inhibiting the PDE3A isoform may lead to the amelioration of excessive vascular SMC growth and decrease the atherosclerosis process [41]. Thus, the abovementioned genes are mainly involved in the regulation of the tricarboxylic acid cycle and respiratory transport chain in terms of energy supply and maintain the normal function of vascular SMC. Finally, CACNB2, KCNAB2, and TIMM22 encode subunits that participate in dysfunctional voltage-gated channels that may be associated with arrhythmia events rather than aggravated heart failure $[42,43]$. Thus, these are factors that are associated with the development of heart failure.

In addition, Table 1 shows us the shared pathway that occurs in both postischemic HF with or without T2DM. Most of the pathways are related to metabolism, such as the following: the citrate cycle (TCA cycle); butanoate, carbon, pyruvate, and 2-oxocarboxylic acid metabolism; and valine, leucine, isoleucine, and fatty acid degradation. Figure 5 shows that it is similar to the pathways of the intersectional coexpression genes and the genes of the module that are enriched within the PPI network. Furthermore, the HIF-1 signaling pathway is a hot topic that researchers focus on. In M1 macrophages, HIF- $1 \alpha$ activates the expression of the
iNOS gene, increasing nitric oxide synthesis, which expands the blood vessels. As such, in hypoxia macrophages, the HIF- $1 \alpha$-pyruvate dehydrogenase kinase (PDK1) axis can induce active glycolysis [44]. In addition, an investigation from Chen et al. [45] suggests that HIF- $1 \alpha$ and FoxO3a show synergistic effects of cardiomyocyte apoptosis under hypoxia, as well as elevated glucose levels. Another pathway, the TGF$\beta$ signaling pathway, is also a popular hot topic. TGF- $\beta$ is a multifunctional cytokine, which can regulate the macrophage phenotype, promote $\mathrm{T}_{\text {reg }}$ cell activation, and reduce adhesion molecule synthesis by endothelial cells that lend a powerful anti-inflammatory effect [46]. Data from the study by Kim et al. show us that the TGF- $\beta$ signaling pathway plays an important role in the regulation of cardiac fibrosis [47]. Lastly, as a classical pathway, the calcium signaling pathway was also found in both the DHF and nDHF patient groups. $\mathrm{Ca}^{2+}$ participates in excitation-contraction coupling, regulating myocardial contraction and diastole. In addition, it also takes part in the regulation of the cardiomyocyte action potential, which plays an essential role in managing heart rhythm $[48,49]$. Thus, regulation disorders of the calcium signaling pathway will lead to heart rate disorders, myocardial contraction, and adrenal dysfunction. The abovementioned pathways affect patients with postischemic heart failure in terms of energy supply, metabolism, inflammation, and myocardial fibrosis.

Compared to HF patients without T2DM, the $N P P B$ coexpression genes were enriched in several other pathways, such as arrhythmogenic right ventricular cardiomyopathy (ARVC), dilated cardiomyopathy, hypertrophic cardiomyopathy (HCM), cardiac muscle contraction, alcoholism, and the PI3K-Akt signaling pathway. The former three are different types of cardiomyopathy, and they mainly affect the morphology and function of ventricular muscle cells, resulting in the deterioration of cardiac function [50]. Alcohol abuse may double the risk of chronic HF compared to those who never had alcohol abuse [51], and the BNP level may increase markedly [34]. In context to the PI3K-Akt signaling pathway, it has been revealed to be involved in the expression level of BNP and in the cardioprotection afforded by BNP infusion $[52,53]$. Thus, these pathways and the genes they enriched would affect the level of BNP and the development of HF. 
Although we use the microarray dataset to help us identify the $N P P B$ coexpression genes and pathways they enriched in postischemic HF patients, either in patients with T2DM or without T2DM, the occurrence and development of HF is complex, and a variety of aspects should be taken in consideration in the management of HF. We hope our findings could give a hand to a deeper understanding of the role and function of the NPPB gene in HF patients and provide aspects for the research and management of $\mathrm{HF}$ in the future.

\section{Conclusions}

The $N P P B$ coexpression genes were used to identify the potential molecular mechanisms of the $N P P B$ gene in DHF and $\mathrm{nDHF}$ patients in this study. Our findings may help elucidate the roles of $N P P B$ and its coexpression genes in postischemic heart failure and serve as a clinical reference for future $\mathrm{HF}$ management. However, further research is required to validate the role of these coexpression genes and pathways.

\section{Abbreviations}

\section{GEO: Gene Expression Omnibus}

GO: Gene Ontology

KEGG: Kyoto Encyclopedia of Genes and Genomes

MCODE: Molecular complex detection

PPI: Protein-protein interaction

BP: $\quad$ Biological processes

CC: $\quad$ Cellular components

MF: $\quad$ Molecular functions

T2DM: $\quad$ Type 2 diabetes mellitus

HF: $\quad$ Heart failure

TCA: Tricarboxylic acid cycle

SMC: $\quad$ Smooth muscle cells.

\section{Data Availability}

The datasets used and/or analyzed during the current study are available from the Gene Expression Omnibus repository (https://www.ncbi.nlm.nih.gov/geo/query/acc.cgi?acc= GSE26887; https://www.ncbi.nlm.nih.gov/geo/query/acc .cgi?acc=GSE5406).

\section{Conflicts of Interest}

The authors declare that they have no competing interests.

\section{Authors' Contributions}

Y.-Z.G. conceived the study, participated in the design, performed the statistical analyses, and drafted the manuscript. R.-X.Y. conceived the study, participated in the design, and helped draft the manuscript. G.-X.D. and P.-F.Z. contributed in formal analysis. C.-X.L. and B.-L.W. helped draft the manuscript. All authors read and approved the final manuscript.

\section{Acknowledgments}

This study was supported by the National Natural Science Foundation of China (No. 81160111).

\section{Supplementary Materials}

Supplementary 1. Figure S1: Protein-protein interaction (PPI) network of NPPB coexpression genes in the DHF group. The red ball represents positive coexpression, while the green ball represents negative coexpression. The thickness of the line represents the strength of the correlation.

Supplementary 2. Figure S2: Modules identified from the PPI network of NPPB coexpression genes in the DHF group. The red ball represents positive coexpression, while the green ball represents negative coexpression. The thickness of the line represents the strength of the correlation. (A-F) Module 1module 6 are identified with a cutoff criterion of MCODE score $>5$.

Supplementary 3. Figure S3: PPI network of NPPB coexpression genes in the nDHF group. The red ball represents positive coexpression, while the green ball represents negative coexpression. The thickness of the line represents the strength of the correlation.

Supplementary 4. Figure S4: Modules identified from the PPI network of NPPB coexpression genes in the $\mathrm{nDHF}$ group. The red ball represents positive coexpression, while the green ball represents negative coexpression. The thickness of the line represents the strength of the correlation. (A-C) Module 1 -module 3 are identified with a cutoff criterion of MCODE score $>5$.

Supplementary 5. Table S1: the GO and KEGG pathways in DHF patients.

Supplementary 6. Table S2: the GO and KEGG pathway in $\mathrm{nDHF}$ patients.

Supplementary 7. Table S3: the GO and KEGG pathways in both groups.

\section{References}

[1] P. Ponikowski, S. D. Anker, K. F. AlHabib et al., "Heart failure: preventing disease and death worldwide," ESC Heart Failure, vol. 1, no. 1, pp. 4-25, 2014.

[2] A. A. Shafie, Y. P. Tan, and C. H. Ng, "Systematic review of economic burden of heart failure," Heart Failure Reviews, vol. 23, no. 1, pp. 131-145, 2017.

[3] M. G. Crespo-Leiro, S. D. Anker, A. P. Maggioni et al., "European Society of Cardiology Heart Failure Long-Term Registry (ESC-HF-LT): 1-year follow-up outcomes and differences across regions," European Journal of Heart Failure, vol. 18, no. 6, pp. 613-625, 2016.

[4] B. Ziaeian and G. C. Fonarow, "Epidemiology and aetiology of heart failure," Nature Reviews. Cardiology, vol. 13, no. 6, pp. 368-378, 2016.

[5] Y. Zheng, S. H. Ley, and F. B. Hu, "Global aetiology and epidemiology of type 2 diabetes mellitus and its complications," Nature Reviews Endocrinology, vol. 14, no. 2, pp. 88-98, 2018. 
[6] R. Rørth, P. S. Jhund, U. M. Mogensen et al., "Risk of incident heart failure in patients with diabetes and asymptomatic left ventricular systolic dysfunction," Diabetes Care, vol. 41, no. 6, pp. 1285-1291, 2018.

[7] P. M. Seferović, M. C. Petrie, G. S. Filippatos et al., “Type 2 diabetes mellitus and heart failure: a position statement from the Heart Failure Association of the European Society of Cardiology," European Journal of Heart Failure, vol. 20, no. 5, pp. 853-872, 2018.

[8] C. W. Yancy, M. Jessup, B. Bozkurt et al., "2017 ACC/AHA/HFSA focused update of the 2013 ACCF/AHA guideline for the management of heart failure: a report of the American College of Cardiology/American Heart Association Task Force on Clinical Practice Guidelines and the Heart Failure Society of America," Journal of the American College of Cardiology, vol. 70, no. 6, pp. 776-803, 2017.

[9] I. S. Thrainsdottir, T. Aspelund, G. Thorgeirsson et al., "The association between glucose abnormalities and heart failure in the population-based Reykjavik study," Diabetes Care, vol. 28, no. 3, pp. 612-616, 2005.

[10] E. Braunwald, "Diabetes, heart failure, and renal dysfunction: the vicious circles," Progress in Cardiovascular Diseases, vol. 62, no. 4, pp. 298-302, 2019.

[11] C. Mueller, K. McDonald, R. A. de Boer et al., "Heart Failure Association of the European Society of Cardiology practical guidance on the use of natriuretic peptide concentrations," European Journal of Heart Failure, vol. 21, no. 6, pp. 715731, 2019.

[12] T. Nishikimi, K. Kuwahara, and K. Nakao, "Current biochemistry, molecular biology, and clinical relevance of natriuretic peptides," Journal of Cardiology, vol. 57, no. 2, pp. 131-140, 2011.

[13] A.-M. Moilanen, J. Rysä, E. Mustonen et al., "Intramyocardial BNP gene delivery improves cardiac function through distinct context-dependent mechanisms," Circulation Heart Failure, vol. 4, no. 4, pp. 483-495, 2011.

[14] S. M. Shaw, J. E. Fildes, C. M. Puchalka, M. Basith, N. Yonan, and S. G. Williams, "BNP directly immunoregulates the innate immune system of cardiac transplant recipients in vitro," Transplant Immunology, vol. 20, no. 3, pp. 199-202, 2009.

[15] S. S. Khanam, E. Choi, J. W. Son et al., "Validation of the MAGGIC (Meta-Analysis Global Group in Chronic Heart Failure) heart failure risk score and the effect of adding natriuretic peptide for predicting mortality after discharge in hospitalized patients with heart failure," PLoS One, vol. 13, no. 11, article e0206380, 2018.

[16] C. Ju, M. Ye, and F. Li, "Plasma brain natriuretic peptide, endothelin-1, and matrix metalloproteinase 9 expression and significance in type 2 diabetes mellitus patients with ischemic heart disease," Medical Science Monitor, vol. 21, pp. 20942099, 2015.

[17] V. Epshteyn, K. Morrison, P. Krishnaswamy et al., "Utility of B-type natriuretic peptide (BNP) as a screen for left ventricular dysfunction in patients with diabetes," Diabetes Care, vol. 26, no. 7, pp. 2081-2087, 2003.

[18] A. Sharma, B. G. Demissei, J. Tromp et al., "A network analysis to compare biomarker profiles in patients with and without diabetes mellitus in acute heart failure," European Journal of Heart Failure, vol. 19, no. 10, pp. 1310-1320, 2017.

[19] I. C. Van der Horst, R. A. De Boer, H. L. Hillege, F. Boomsma, A. A. Voors, and D. J. Van Veldhuisen, "Neurohormonal pro- file of patients with heart failure and diabetes," Netherlands Heart Journal, vol. 18, no. 4, pp. 190-196, 2010.

[20] T. Barrett, S. E. Wilhite, P. Ledoux et al., "NCBI GEO: archive for functional genomics data sets-update," Nucleic Acids Research, vol. 41, no. Database issue, pp. D991-D995, 2013.

[21] N. Li, H. Wu, R. Geng, and Q. Tang, "Identification of core gene biomarkers in patients with diabetic cardiomyopathy," Disease Markers, vol. 2018, Article ID 6025061, 15 pages, 2018.

[22] M. E. Ritchie, D. Diyagama, J. Neilson et al., "Empirical array quality weights in the analysis of microarray data," BMC Bioinformatics, vol. 7, no. 1, p. 261, 2006.

[23] D. W. Huang, B. T. Sherman, and R. A. Lempicki, "Systematic and integrative analysis of large gene lists using DAVID bioinformatics resources," Nature Protocols, vol. 4, no. 1, pp. 44-57, 2009.

[24] M. Kanehisa, M. Furumichi, M. Tanabe, Y. Sato, and K. Morishima, "KEGG: new perspectives on genomes, pathways, diseases and drugs," Nucleic Acids Research, vol. 45, no. D1, pp. D353-D361, 2017.

[25] I. Hulsegge, A. Kommadath, and M. A. Smits, "Globaltest and GOEAST: two different approaches for Gene Ontology analysis," BMC Proceedings, vol. 3, Supplement 4, p. S10, 2009.

[26] D. Szklarczyk, A. Franceschini, S. Wyder et al., "STRING v10: protein-protein interaction networks, integrated over the tree of life," Nucleic Acids Research, vol. 43, no. D1, pp. D447D452, 2015.

[27] P. Shannon, A. Markiel, O. Ozier et al., "Cytoscape: a software environment for integrated models of biomolecular interaction networks," Genome Research, vol. 13, no. 11, pp. 24982504, 2003.

[28] S. Fu, P. Ping, F. Wang, and L. Luo, "Synthesis, secretion, function, metabolism and application of natriuretic peptides in heart failure," Journal of Biological Engineering, vol. 12, no. 1, p. 2, 2018.

[29] K. Zaugg, Y. Yao, P. T. Reilly et al., "Carnitine palmitoyltransferase 1C promotes cell survival and tumor growth under conditions of metabolic stress," Genes \& Development, vol. 25, no. 10, pp. 1041-1051, 2011.

[30] R. Klose, A. Prinz, F. Tetzlaff et al., "Loss of the serine protease HTRA1 impairs smooth muscle cells maturation," Scientific Reports, vol. 9, no. 1, p. 18224, 2019.

[31] J. J. L. Williams, N. Alotaiq, W. Mullen et al., "Interaction of suppressor of cytokine signalling 3 with cavin-1 links SOCS3 function and cavin-1 stability," Nature Communications, vol. 9, no. 1, p. 168, 2018.

[32] K. Dhar, K. Rakesh, D. Pankajakshan, and D. K. Agrawal, "SOCS3 promotor hypermethylation and STAT3-NF- $\kappa$ B interaction downregulate SOCS3 expression in human coronary artery smooth muscle cells," American Journal of Physiology Heart and Circulatory Physiology, vol. 304, no. 6, pp. H776-H785, 2013.

[33] J. Zhang, G. Wu, and H. Dai, "The matricellular protein CCN1 regulates TNF- $\alpha$ induced vascular endothelial cell apoptosis," Cell Biology International, vol. 40, no. 1, pp. 1-6, 2016.

[34] R. Stark, A. Reichenbach, and Z. B. Andrews, "Hypothalamic carnitine metabolism integrates nutrient and hormonal feedback to regulate energy homeostasis," Molecular and Cellular Endocrinology, vol. 418, Part 1, pp. 9-16, 2015.

[35] S. B. Thomsen, C. N. Rathcke, B. Zerahn, and H. Vestergaard, "Increased levels of the calcification marker matrix Gla protein and the inflammatory markers YKL-40 and CRP in patients 
with type 2 diabetes and ischemic heart disease," Cardiovascular Diabetology, vol. 9, no. 1, p. 86, 2010.

[36] G. Arderiu, S. Espinosa, E. Pena, R. Aledo, and L. Badimon, "PAR2-SMAD3 in microvascular endothelial cells is indispensable for vascular stability via tissue factor signaling," Journal of Molecular Cell Biology, vol. 8, no. 3, pp. 255-270, 2016.

[37] S. M. Jones, A. Mann, K. Conrad et al., "PAR2 (protease-activated receptor 2) deficiency attenuates atherosclerosis in mice," Arteriosclerosis, Thrombosis, and Vascular Biology, vol. 38, no. 6, pp. 1271-1282, 2018.

[38] A. G. Reidenbach, Z. A. Kemmerer, D. Aydin et al., "Conserved lipid and small-molecule modulation of COQ8 reveals regulation of the ancient kinase-like UbiB family," Cell Chemical Biology, vol. 25, no. 2, pp. 154-165.e11, 2018.

[39] T. Lobo-Jarne and C. Ugalde, "Respiratory chain supercomplexes: structures, function and biogenesis," Seminars in Cell \& Developmental Biology, vol. 76, pp. 179-190, 2018.

[40] C. Yan, "Cyclic nucleotide phosphodiesterase 1 and vascular aging," Clinical Science, vol. 129, no. 12, pp. 1077-1081, 2015.

[41] N. Begum, W. Shen, and V. Manganiello, "Role of PDE3A in regulation of cell cycle progression in mouse vascular smooth muscle cells and oocytes: implications in cardiovascular diseases and infertility," Current Opinion in Pharmacology, vol. 11, no. 6, pp. 725-729, 2011.

[42] P. Kovermann, K. N. Truscott, B. Guiard et al., "Tim22, the essential core of the mitochondrial protein insertion complex, forms a voltage-activated and signal-gated channel," Molecular Cell, vol. 9, no. 2, pp. 363-373, 2002.

[43] D. Schultz, M. Litt, L. Smith, M. Thayer, and K. McCormack, "Localization of two potassium channel beta subunit genes, KCNA1B and KCNA2B," Genomics, vol. 31, no. 3, pp. 389391, 1996.

[44] H. Abe, H. Semba, and N. Takeda, "The roles of hypoxia signaling in the pathogenesis of cardiovascular diseases," Journal of Atherosclerosis and Thrombosis, vol. 24, no. 9, pp. 884-894, 2017.

[45] Y.-F. Chen, S. Pandey, C. H. Day et al., "Synergistic effect of HIF- $1 \alpha$ and FoxO3a trigger cardiomyocyte apoptosis under hyperglycemic ischemia condition," Journal of Cellular Physiology, vol. 233, no. 4, pp. 3660-3671, 2018.

[46] N. G. Frangogiannis, "The inflammatory response in myocardial injury, repair, and remodelling," Nature Reviews. Cardiology, vol. 11, no. 5, pp. 255-265, 2014.

[47] J. Kim, J. Kim, S. H. Lee et al., "Cytokine-like 1 regulates cardiac fibrosis via modulation of TGF- $\beta$ signaling," PLoS One, vol. 11, no. 11, article e0166480, 2016.

[48] Y. K. Tham, B. C. Bernardo, J. Y. Y. Ooi, K. L. Weeks, and J. R. McMullen, "Pathophysiology of cardiac hypertrophy and heart failure: signaling pathways and novel therapeutic targets," Archives of Toxicology, vol. 89, no. 9, pp. 1401-1438, 2015.

[49] G. Gilbert, K. Demydenko, E. Dries et al., "Calcium signaling in cardiomyocyte function," Cold Spring Harbor Perspectives in Biology, vol. 12, no. 3, p. a035428, 2020.

[50] E. Arbustini, N. Narula, L. Tavazzi et al., "The MOGE(S) classification of cardiomyopathy for clinicians," Journal of the American College of Cardiology, vol. 64, no. 3, pp. 304-318, 2014.

[51] I. R. Whitman, V. Agarwal, G. Nah et al., "Alcohol abuse and cardiac disease," Journal of the American College of Cardiology, vol. 69, no. 1, pp. 13-24, 2017.
[52] G. Hu, X. Huang, K. Zhang, H. Jiang, and X. Hu, “Anti-inflammatory effect of B-type natriuretic peptide postconditioning during myocardial ischemia-reperfusion: involvement of PI3K/Akt signaling pathway," Inflammation, vol. 37, no. 5, pp. 1669-1674, 2014.

[53] L. Breivik, A. Jensen, S. Guvåg et al., "B-type natriuretic peptide expression and cardioprotection is regulated by Akt dependent signaling at early reperfusion," Peptides, vol. 66, pp. 43-50, 2015. 